Classification

Physics Abstracts

$61.30 \mathrm{~J}-47.15$

\title{
Anomalies de l'écoulement läminaire d'un smectique A autour d'un obstacle cylindrique
}

\author{
P. Oswald \\ Laboratoire de Physique des Solides (*), Université Paris-Sud, 91405 Orsay, France
}

(Reçu le 13 janvier 1983, accepté le 1er mars 1983)

\begin{abstract}
Résumé. - En cisaillant un échantillon homéotrope de smectique A, nous observons une rigidification importante de la partie centrale de l'écoulement. Cet effet, sans doute lié à la présence de défauts invisibles au microscope, subsiste en présence d'un fil cylindrique collé sur l'une des faces de l'échantillon. De part et d'autre de cet obstacle s'étendent deux régions statiques raccordées à la partie centrale de l'écoulement au travers d'une couche mince de perméation. Une évaluation de la puissance dissipée nous permet d'estimer la longueur de perméation $\chi^{-1}=\sqrt{\eta \lambda_{\mathrm{p}}}$ pour la première fois introduite par de Gennes. Nous montrons enfin qu'une dilatation sous cisaillement conduit à un réseau de paraboles focales asymétrique par rapport à l'obstacle.
\end{abstract}

\begin{abstract}
When shearing a homeotropic sample of smectic A, a strong rigidification of the central part of the flow has been observed. This effect has probably to do with the presence of defects that are not visible under the microscope. It subsists when a cylindrical thread is glued on one side of the sample. Two static regions then spread out on both sides of this obstacle. They are connected to the central part of the flow through a thin layer of permeation. An estimate of the dissipation gives us a rough value of the permeation length, first introduced by de Gennes : $\chi^{-1}=\sqrt{\eta \lambda_{\mathrm{p}}}$. Lastly we show that a dilation under shear leads to a lattice of focal parabola, which is asymmetrical with respect to the obstacle.
\end{abstract}

Un cristal liquide smectique A présente l'originalité d'être un liquide à deux dimensions dans le plan de ses couches et un solide dans la direction perpendiculaire. Ce double caractère est à l'origine d'effets hydrodynamiques remarquables.

Les premières tentatives visant à les décrire sont celles de P. G. de Gennes et du Groupe d'Orsay des Cristaux liquides [1].

Le cas du cisaillement perpendiculaire aux couches a été successivement étudié par C. E. Williams puis par J. Marignan [2]. Ces travaux ont montré le rôle important des défauts (coniques focales) dans l'écoulement du smectique. Cet article décrit le comportement d'un smectique soumis à un cisaillement parallèle aux couches. Il montre les anomalies de l'écoulement de Couette simple que nous généralisons au cas d'un écoulement autour d'obstacles cylindriques de diffé-

$\left(^{*}\right)$ Laboratoire associé au CNRS. 
rents diamètres. Nous décrivons ensuite le comportement des défauts créés par dilatation au cours du cisaillement. Nous terminons par quelques remarques de nature théorique sur la couche limite de perméation et sur le rôle éventuel des défauts dans cette géométrie d'écoulement.

\section{Dispositif expérimental.}

La cellule de cisaillement utilisée a déjà été décrite dans un article antérieur [3]. Elle permet de cisailler avec une grande régularité un cristal liquide placé entre deux lames de verre parallèles. Cette cellule a été conçue pour les observations au microscope polarisant. Les lames de verres sont traitées au silane polymérisé pour assurer un bon ancrage homéotrope. L'obstacle utilisé est un fil cylindrique en nylon qui est collé à ses extrémités sur la lame inférieure que l'on maintient immobile. La lame supérieure effectue un mouvement de translation dans la direction perpendiculaire à l'axe du fil. Sa vitesse $V$ peut várier de 1 à $70 \mu \mathrm{m} / \mathrm{s}$. L'épaisseur des échantillons est de $280 \mu \mathrm{m}$.

Le produit étudié est le $8 \mathrm{CB}$ (4-n-octyl-4'-cyanobiphényle) qui a une phase Sm A entre $21^{\circ} \mathrm{C}$ et $33^{\circ} \mathrm{C}$ et est nématique au-dessus de $33^{\circ} \mathrm{C}$.

\section{Les anomalies de l'écoulement laminaire.}

Ce paragraphe décrit le cisaillement continu d'échantillons homéotropes. Qu'il y ait ou non un obstacle, l'extinction reste parfaite entre polariseurs croisés au cours du mouvement. L'écoulement est stable à l'échelle du microscope optique. Ceci exige une grande stabilité de la cellule de cisaillement et un bon parallélisme des lames dont l'angle n'excède pas $10^{-4} \mathrm{rad}$. Pour déterminer le profil des vitesses nous suivons dans leur mouvement de fines poussières toujours présentes dans les échantillons. Leur répartition aléatoire et la difficulté de les positionner dans l'épaisseur avec précision rendent nos résultats approximatifs. La figure 1 représente le profil des vitesses

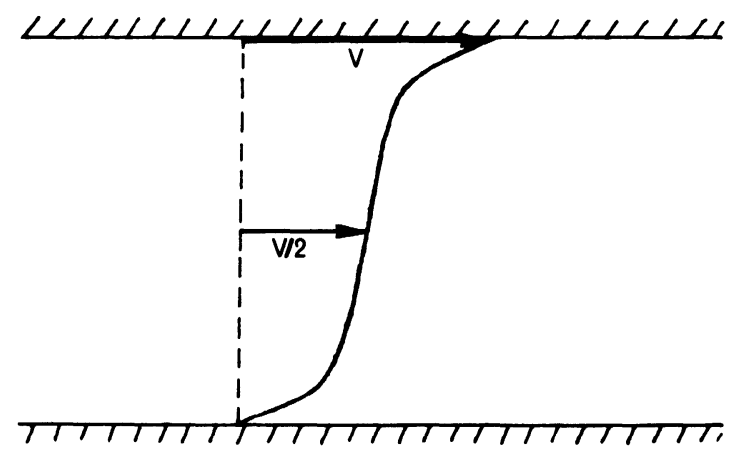

Fig. 1. - Profil des vitesses en cisaillement simple parallèle aux couches.

[Velocity profile under simple shear parallel to the layers.]

pour un cisaillement sans obstacle. Nous observons un écart important au profil linéaire et une rigidification très nette du centre de l'échantillon. Cette région se caractérise par un faible taux de cisaillement. Elle est animée d'une vitesse moyenne moitié de la vitesse $V$ de la lame supérieure. Au voisinage des lames s'étendent deux zones épaisses de $30 \mu \mathrm{m}$ environ et soumises à un fort gradient des vitesses. L'allure de ce profil dépend peu de la vitesse de cisaillement $V$ et la rigidification subsiste au très faible taux de cisaillement $(V \sim 1 \mu \mathrm{m} / \mathrm{s})$. 
La présence d'un obstacle modifie ce comportement. Nous pouvons alors distinguer dans l'écoulement trois régions (Fig. 2) :

- La première, caractérisée par un fort gradient des vitesses, s'étend sur une profondeur $h$ qui croît avec le diamètre $d$ de l'obstacle. La vitesse varie de $V$ à $V^{\prime}$ dans cette région.

- Lui succède une région de faible vorticité qui s'étend jusqu'au sommet de l'obstacle et où la vitesse est quasiment constante et égale à $V^{\prime}$.

- Une dernière zone statique s'étend de part et d'autre de l'obstacle. La limite qui la sépare de la précédente et dans laquelle s'annule la vitesse n'a pu être résolue au microscope.

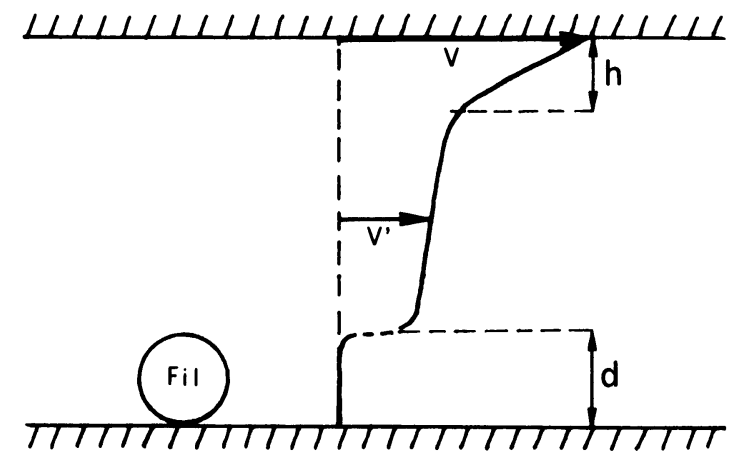

Fig. 2. - Profil des vitesses sous cisaillement en présence d'un fil cylindrique.

[Velocity profile under shear in presence of a cylindrical thread.]

Voici à titre indicatif les ordres de grandeur de $h$ et du rapport $V / V^{\prime}$ pour diverses valeurs du diamètre $d$ du fil. Dans tous les cas l'épaisseur totale de l'échantillon est de $280 \mu \mathrm{m}$.

\begin{tabular}{|c|c|c|}
\hline$d( \pm 5 \mu \mathrm{m})$ & $h( \pm 15 \mu \mathrm{m})$ & $V / V^{\prime}( \pm 0,5)$ \\
\hline 20 & 30 & 2,5 \\
40 & 40 & 3 \\
80 & 60 & 4,5 \\
110 & 100 & 7 \\
\hline
\end{tabular}

Pour compléter nos résultats nous allons décrire l'effet d'une dilatation au cours du cisaillement. Ceci fait l'objet du paragraphe suivant.

\section{Instabilités.}

Nous savons que sous l'effet d'une dilatation normale aux couches se développe rapidement un réseau de paraboles focales.

En statique ce réseau est désordonné [4].

Sous cisaillement il apparaît mieux ordonné. Sans obstacle les domaines focaux qui le composent occupent la partie médiane de.l'échantillon qui devient encore plus rigide que lors de l'écoulement de Couette simple. Ces défauts se déplacent en bloc à une vitesse moitié de celle de la lame mobile. Une description détaillée et une première analyse théorique de ces phénomènes sont données respectivement dans [3] et [5]. 
En présence d'un obstacle et sous cisaillement, la situation devient plus complexe. La nucléation du réseau au moment de la dilatation reste inchangée ainsi que la taille moyenne des défauts qui ne dépend que de l'épaisseur totale de l'échantillon. Par contre l'évolution future de ce réseau dépend du diamètre du fil utilisé.

L'introduction d'un fil de $20 \mu \mathrm{m}$ de diamètre n'apporte aucun changement important. Les défauts toujours centrés dans l'épaisseur se déplacent rigidement à une vitesse voisine de $0,4 \mathrm{~V}$. Ils passent à l'aplomb de l'obstacle sans modification.

Avec un fil de $40 \mu \mathrm{m}$ la texture est entraînée à une vitesse plus petite, proche de $0,3 \mathrm{~V}$. Un effet nouveau survient au voisinage du fil. Les défauts en provenance de l'amont s'« annihilent " à son passage pour réapparaître à l'aval sous forme d'un nouveau réseau très bien ordonné dont le paramètre $a$ est toujours supérieur à celui de la texture initiale (Fig. 3a). D'autre part l'observation des points de rencontre des paraboles a montré qu'elles occupent une épaisseur $t$ plus grande que dans le reste de l'échantillon (Fig. $3 b$ ). La mesure de $a$ et $t$ de part et d'autre du fil permet de connaitre la distance focale $f$ des paraboles $\left(a^{2}=8 f t\right)$ et de montrer qu'elle reste invariante après passage à l'aplomb de l'obstacle. L'effet principal du fil est donc de favoriser un réarrangement des domaines focaux et de conduire à une diminution globale de l'énergie élastique du réseau.

Avec un fil de $80 \mu \mathrm{m}$ apparaît un décentrage visible des domaines paraboliques (Fig. $4 a$ ). Cet effet survient au moment de la nucléation sous cisaillement. Les défauts du réseau amont sont décentrés vers le bas et ne peuvent franchir l'obstacle. Ils restent immobiles. A l'aval les défauts

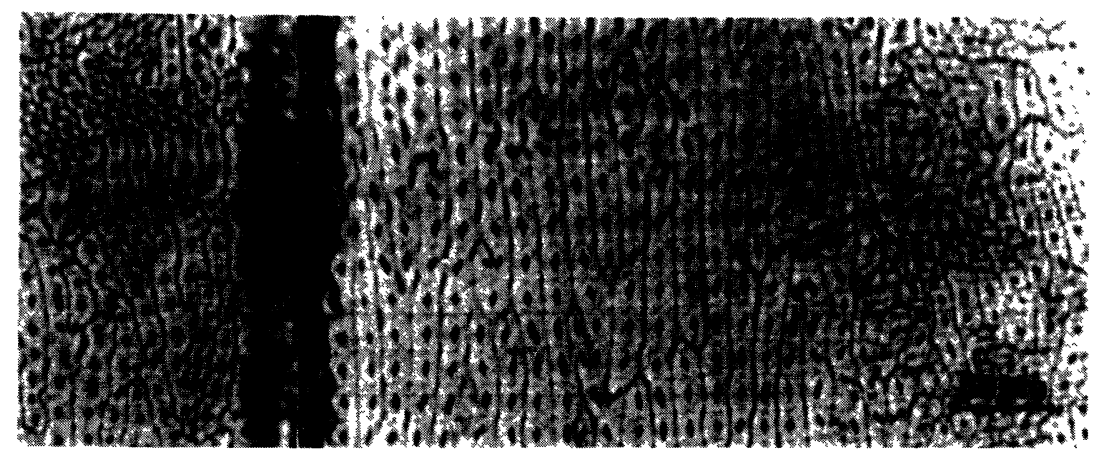

a)

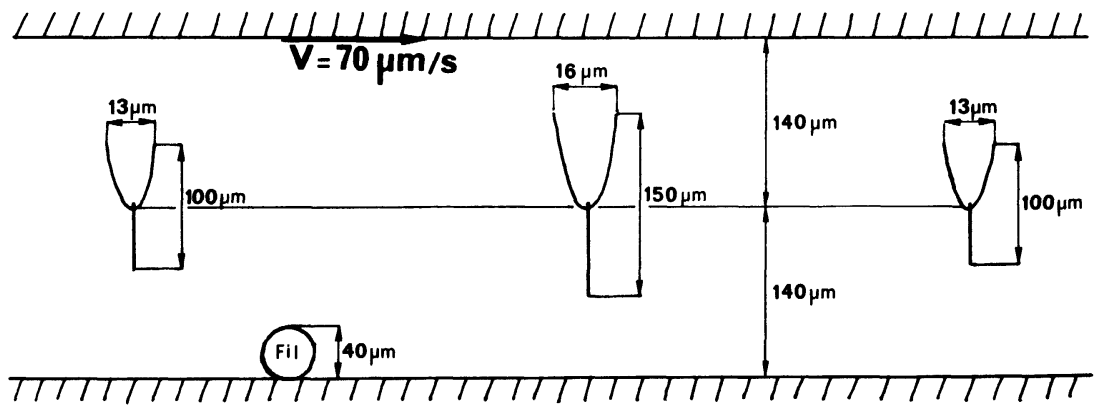

b)

Fig. 3. - $a$ : Aspect de la texture obtenue après cisaillement en présence d'un fil de $40 \mu \mathrm{m}$ de diamètre, la dilatation (de l'ordre du micron) étant maintenue. A l'aval est apparu un réseau très bien ordonné de domaines paraboliques plus gros (Nicols/croisés). $b$ : Disposition des paraboles focales au sein de l'échantillon.

[ $a$ : Aspect of the texture obtained under shear in presence of a $40 \mu \mathrm{m}$ diameter thread, the dilation (about one micron) being kept constant. A very well ordered lattice of larger parabolic domains appeared downstream (Crossed Nicols). $b$ : Arrangement of the focal parabola inside the sample.] 


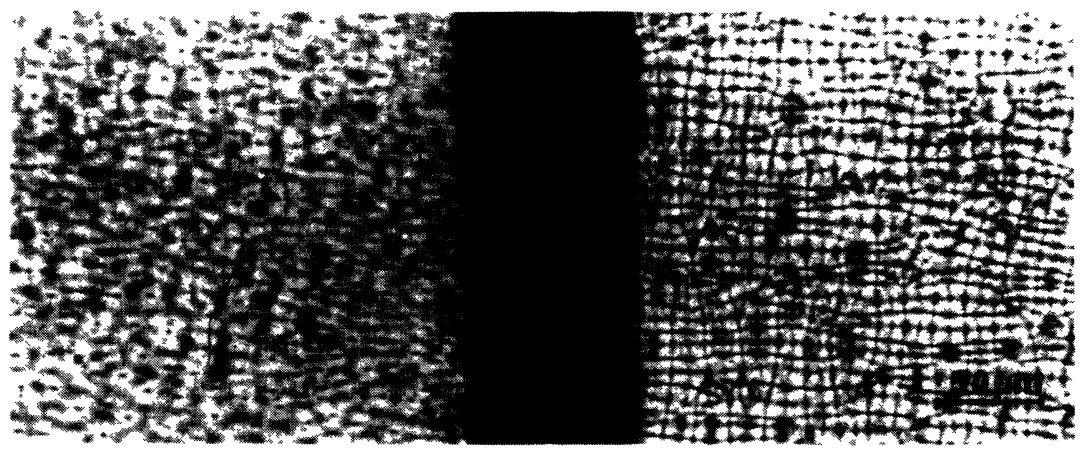

a)

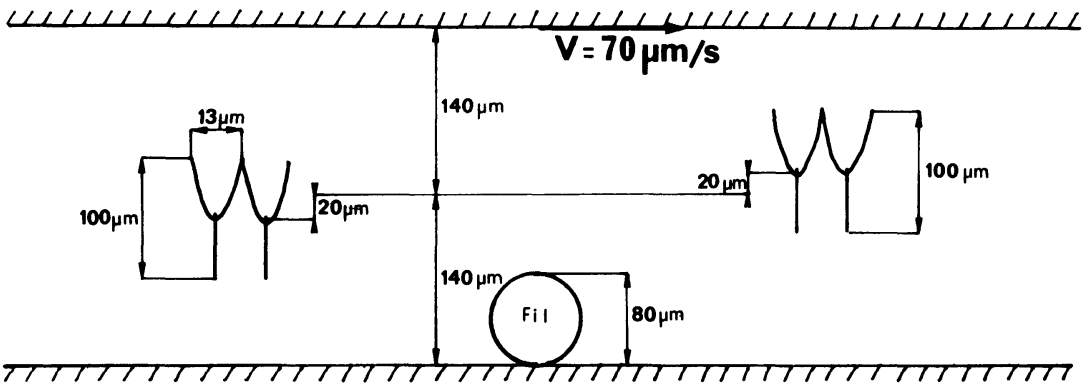

b)

Fig. 4. - $a$ : Aspect du réseau de paraboles focales juste après sa nucléation sous cisaillement en présence d'un fil de $80 \mu \mathrm{m}$ de diamètre. Le décentrage des domaines focaux est très net : à l'amont de l'obstacle sont visibles les points de rencontre des paraboles tandis qu'à l'aval la mise au point apparaît sur le cour des domaines (Nicols croisés). $b$ : Disposition des paraboles focales au sein de l'échantillon.

[ $a:$ Aspect of the focal parabola lattice just after its nucleation under shear in presence of a $80 \mu \mathrm{m}$ diameter thread. The focal domains are clearly out of the centre; up-stream from the obstacle, the parabola-intersection points are visible while down-stream, the focus is on the core of the domains (Crossed Nicols). $b$ : Arrangement of the focal parabola inside the sample.]

sont décentrés vers le haut et sont entraînés par l'écoulement à une vitesse voisine de $0,2 V$ (Fig. $4 b$ ). Au niveau du fil apparaît comme précédemment un réseau bien ordonné de défauts plus gros.

Avec un fil de $110 \mu \mathrm{m}$ la situation est sensiblement la même, et le décentrage s'accentue légèrement. Néanmoins le cisaillement devient très destructeur et conduit rapidement à un échantillon de nouveau homéotrope.

Les effets que l'on vient de décrire sont reproductibles. Ils dépendent néanmoins beaucoup de paramètres difficilement contrôlables, comme la valeur de la dilatation imposée (de l'ordre du micron dans ce qui précède) et l'état de surface des lames. La force de l'ancrage dont l'effet est discuté dans [3] peut jouer un rôle important lors de la nucléation des paraboles focales et dans le contenu en défauts, invisibles au microscope, des échantillons homéotropes du départ. L'importance de ce dernier point est soulignée dans le paragraphe suivant.

\section{Eléments d'interprétation.}

Le phénomène le plus surprenant est sans aucun doute la rigidification centrale des échantillons homéotropes sous cisaillement simple. Cet effet s'accentue sous dilatation en présence d'un réseau de paraboles focales. C'est pourquoi nous proposons de l'attribuer à la présence de défauts micro- 
scopiques invisibles au microscope ; ce peut être des dislocations coin ou vis ou des ondulations. Nous n'avons jusqu'à maintenant aucune preuve expérimentale de cette hypothèse. Nous pensons néanmoins que les défauts créent, sous cisaillement, un écoulement de perméation transverse qui rigidifie la partie centrale de l'échantillon. Notons à ce sujet que cet effet subsiste au voisinage de la transition vers la phase nématique où la perméation devient importante.

La présence d'un obstacle ajoute en complexité aux phénomènes décrits précédemment. Néanmoins l'expérience montre clairement l'existence de 2 régions statiques qui s'étendent de part et d'autre de l'obstacle et qui se raccordent à la partie centrale de l'écoulement au travers d'une couche mince de vorticité. Le principe du calcul de la distribution de vitesse dans cette couche limite a été donné par P. G. de Gennes [1]. On peut montrer que l'énergie dissipée par perméation et friction visqueuse dans cette couche mince s'écrit :

$$
\phi^{\prime}=\frac{1}{\sqrt{\pi}} \eta V^{\prime 2} \sqrt{\frac{L}{\chi^{-1}}} .
$$

$L$ désigne la dimension linéaire (dans le plan des couches) de l'échantillon et $\chi^{-1}=\sqrt{\eta \lambda_{p}}$ est la longueur de perméation. Ce calcul suppose constante et égale à $V^{\prime}$ la vitesse dans la partie centrale de l'échantillon. Cette énergie doit être égale à celle qui est dissipée dans l'écoulement de Couette localisé au voisinage de la lame supérieure et qui vaut :

$$
\phi^{\prime \prime}=\eta \frac{\left(V-V^{\prime}\right)^{2}}{h^{2}} L
$$

L'égalité $\phi^{\prime}=\phi^{\prime \prime}$, qui exprime que les forces qui s'exercent sur les lames inférieure et supérieure sont égales et opposées, conduit à la relation suivante :

$$
\chi^{-1}=\frac{1}{\pi} \frac{1}{\left(\frac{V}{V^{\prime}}-1\right)^{2}} \frac{h^{2}}{L}
$$

Nous avons donné dans le paragraphe 2 quelques couples de valeurs $\left(h, V / V^{\prime}\right)$. Ils satisfont à cette relation et conduisent pour le $8 \mathrm{CB}$ à la température ambiante à $\chi^{-1} \sim 70 \AA$. Cette valeur est en accord avec celle obtenue lors des expériences de lubrification [6].

\section{Conclusion.}

Cette expérience met en évidence l'existence d'un profil des vitesses du type "bouchon » en cisaillement simple parallèle aux couches smectiques. Ce résultat est compatible avec un comportement non newtonien observé d'abord par M. G. Kim et al. [7] puis par R. G. Horn et M. Kléman [8] dans cette géométrie d'écoulement.

Il est probable que les défauts jouent ici un rôle important comme c'est le cas dans d'autres situations expérimentales [9].

\section{Remerciements.}

Je remefcie M. M. Kléman pour ses multiples conseils et suggestions tout au long de ce travail. 


\section{Bibliographie}

[1] De Gennes, P. G., Phys. Fluids 17 no 9 (1974) 1645.

Orsay Group on Liquid Crystals, J. Physique Colloq. 36 (1975) C1-305.

[2] Williams, C. E., Kléman, M., J. Physique Colloq. 36 (1975) C1-315.

Marignan, J., Maler, G., Parodi, O., Ann. Phys. 3 (1978) 221.

[3] Oswald, P., Béhar, J., Kléman, M., Philos. Mag. A 46 no 6 (1982) 899.

[4] Rosenblatt, Ch. S., Pindak, R., Clark, N. A., Meyer, R. B., J. Physique 38 (1977) 1105.

[5] Oswald, P., Ben-Abraham, S. I., J. Physique 43 (1982) 1193.

Ben-Abraham, S. I., Oswald, P., à paraître dans Mol. Cryst. Liq. Cryst.

[6] Oswald, P., Kléman, M., C.R. Hebd. Sean. Acad. Sci. 294 (1982) Série II, 1057 ; Version anglaise J. Physique Lett. 43 (1982) L-411.

[7] Kim, M. G., PARK, S., CoOper, Sr. M., LetCher, S. V., Mol. Cryst. Liq. Cryst. 36 (1976) 143.

[8] Horn, R. G., Kléman, M., Ann. Phys. 3 (1978) 229.

[9] Voir par exemple l'article de revue de FrIEDEL, J., J. Physique Colloq. 40 (1979) C3-45. 